[6] R. Hunt and R. L. Wheeden, On the boundary values of harmonic functions, Trans. Amer. Math. Soc. 132 (1968), pp. 307-322.

[7] -, - Positive harmonic functions on Iipschitz domains, ibid. 147 (1970), pp. 507-527.

[8] L. Hörmander, $L^{p}$ estimates for (pluri-)subharmonic functions, Math. Scand. 20 (1967), pp. 65-78.

[9] J. K. Kemper, A boundary Harnack principle for Lipschitz domains and the principle of positive singularities, Comm. Pure Appl. Math. 25 (1972), pp. 247-255.

[10] U. Küran, $n$-dimensional extensions of theorems on conjugate functions, Proe. London Math. Soc. (3) 15 (1965), pp. 713-730.

[11] E. M. Stein, Singular integrals and differentiability properties of functions, Princeton University Press, Princeton, New Jersey 1970.

[12] E. M. Stein and G. Weiss, Introduction to Fourier analysis on Euclidean spaces, Princeton University Press, Princeton, Now Jersey 1971.

\section{On weakly* conditionally compact dynamical systems}

by

W. SZLENK (Warszawa)

1. Tet $(X, \varphi)$ be a topological dynamical system, i.e. $X$ is a compact metric space and $\varphi: X \rightarrow X$ is a continuous mapping. Denote by $C(X)$ the space of all continuous, real (or complex) valued functions on $X$, and let $U_{\varphi}$ be an operator defined as follows: $U_{\varphi} f=f \circ \varphi, f \in O(X)$.

A sequence $\left(f_{n}\right)$ of elements of a Banach space $E$ is said to be weakly* conditionally compact if for every sequence of positive integers $\left(n_{k}\right)$ there is a subsequence $\left(n_{k_{i}}\right)$ such that for every linear continuous functional $\Phi \in Z^{*}$ the sequence of scalars $\left(\Phi\left(f_{n_{b_{i}}}\right)\right)$ is convergent. In the case of $E=C(X)$ it means that the sequence $\left(f_{n_{k_{i}}}(x)\right)$ is pointwise convergent (not necessarily to a continuous function).

If for every sequence $\left(n_{k}\right)$ there exists a subsequence $\left(n_{k_{i}}\right)$ and an element $f \in E$ such that $\left(f_{r_{i}}\right)$ is weakly convergent to $f$, then the sequence $\left(f_{n}\right)$ is said to be weakly conditionally compact.

DentnTtron. A system $(X, \varphi)$ is said to be weakly* [weakly] conditionally compact if for every $f \in C(X)$ the sequence $\left(U^{n} f\right)$ is weakly* [weakly] conditionally compact. For brevity, we shall call these systems $w^{*} c c[w c c]$ systems.

The aim of the paper is to stady some spectral properties, the strict ergodicity (under some additional assumptions) and the sequence entropy of $\mathrm{w}^{*} \mathrm{cc}$ systems.

In view of Rosenthal's theorem [8] for every $f \in O(X)$ there are two possibilities:

(1) The sequence $\left(U^{n} f\right)$ contains a subsequence $\left(U^{n_{k f}} f\right)$ such that for some $c>0$ and for every sequence of numbers (real or eomplex) $a_{0}, \ldots, a_{m-1}$ the following inequality holds:

$$
\sup _{x \in X}\left|\sum_{k=0}^{m-1} a_{k} U^{n_{k}} f(x)\right| \geqslant c \sum_{k=0}^{m-I}\left|a_{k}\right| .
$$

(2) The sequence $\left(U^{n} f\right)$ is $w^{*} c c$. 
If a system $(X, \varphi)$ is a $w^{*} c c$ system, then the first possibility does not occur for any $f \in C(X)$. It means that $\varphi$ "mixes" the points in $X$ very slowly for every sequence of moments $\left(n_{k}\right)$.

The wce systems have been studied in [1] and in [10].

ExAMPLEs. (i) Every homeomorphism of an interval is a $\mathrm{w}^{*} \mathrm{cos}$ system (and it is not a wcc system, except the identity) - this follows easily from Proposition 1.

(ii) Every homeomorphism of the circle $S^{1}$ is a $w^{*} c e$ system. The homeomorphism is a wce system if and only if it is topologically equivalent to a rotation.

Proof. It is enough to consider the case where $\varphi$ preserves orientation. If $\varphi$ has a periodic point, then the problem can be reduced to the case (i). Suppose $\varphi$ has no periodic points. Then either $\varphi$ is conjugate to a rotation and evidently the system is a wec system, or $\varphi$ is a so called Denjoy homeomorphism. The set of all non-wandering points is then a Cantor set $\Delta$ in $S^{1}$. For every sequence $\left(n_{k}\right)$ there exists a subsequence $\left(n_{k_{i}}\right)$ such that $\left(\varphi^{n_{k}}(x)\right)$ is convergent for every end-point of each juterval of $S^{1}-\Delta$. Hence we easily conclude that $\left(\varphi^{n_{f_{i}}}(x)\right)$ is convergent for every $x \in S^{1}$, which completes the proof (see Proposition 1).

(iii) Every Morse-Smale system (for the definition see [5] or [11]) is a $\mathrm{w}^{*} \mathrm{cc}$ system.

(iv) Let $X=T^{2}$ be the two-dimensional torus, let $\varphi_{0}: S^{1} \rightarrow S^{1}$ be a homeomorphism of the circle $S^{1}$ and let $k: \mathcal{S}^{1} \rightarrow \mathcal{S}^{1}$ be a continuous mapping. The map $\varphi\left(x^{1}, x^{2}\right)=\left(\varphi_{0}\left(x^{1}\right), x_{2}+f\left(x^{1}\right)\right)$ is called the skev rotation. Let $\hat{\varphi}$ be a lift of $\varphi$ to the plane $R^{2}$, and let $Q$ be a unit square in $\boldsymbol{R}^{2}$. If sup diam $\hat{\varphi}^{n}(Q)$ is finite, then $\left(T^{2}, \varphi\right)$ is a $w^{*}$ cc system. It easily follows from the Gottschalk-Heldlund lemma that for a continuous function $f$ on $S^{1}$ if $f\left(x^{1}\right)+f\left(\varphi_{0}\left(x^{1}\right)\right) \ldots f\left(\varphi_{0}^{n-1}\left(x^{1}\right)\right)$ is uniformly bounded, then there exists a continuous function $g$ such that $g\left(\varphi_{0}\left(x^{1}\right)\right)-g\left(x^{1}\right)=f\left(x^{1}\right)$.

Probably there are some w.ce skew rotations on the torus $T^{2}$ for which $\sup \operatorname{diam} \varphi^{n}(Q)=+\infty$.

2. Proposition 1. A system $(X, \varphi)$ is $\mathrm{w}^{*} \mathrm{ce}$ iff for every sequence of positive integers $\left(n_{k}\right)$ there exists a subsequence $\left(n_{k_{i}}\right)$ such that the sequence $\left(\varphi^{n_{k_{i}}}(x)\right)$ is convergent for every $x \in X$.

$A$ system $(X, \varphi)$ is wco iff every cluster point $\psi$ of the set $\left\{\varphi^{n}\right\}$ in pointwise topology is continuous.

The proof is quite elementary, and so we omit it.

THEOREM 1. Let $\mu$ be an invariant ergadic probabilistic measure for $a \mathrm{w}^{*} \mathrm{cc}$ system $(X, \varphi)$. Then the metric system $(X, B, \mu, \varphi)(B$ ofield of Borel sets) has a discrete spectrum. Moreover, the eigenfunctions of $U_{\varphi}$ are of the first Baire olass.
Proof. Let $\|\cdot\|$ denote the norm in the space $C(\bar{X})$, and let $\|\cdot\|_{2}$ denote the norm in the space $I^{2}(X, B, \mu)$. By assumption every sequence $\left(U_{\phi}^{n} f\right)$, $f \in C(X)$, is a bounded sequence of functions, conditionally compact in the topology of pointwise convergence. Therefore $\left(U_{\varphi}^{n} f\right)$ is conditionally compact in the space $L^{2}(X, B, \mu)$. Since $C(X)$ is dense in $L^{2}(X, B, \mu)$, the sequence $\left(U_{q}^{n} f\right)$ is conditionally compact for every $f \in L^{2}(X, B, \mu)$. Thus by Kushnirenko's theorem [4] the spectrum of $U_{\varphi}$ in $L^{2}(X, B, \mu)$ is discrete. Denote by $\left(f_{n}\right)$ the sequence of all eigenfunctions of $U_{q}: U_{q} f_{n}$ $=\lambda_{n} f_{n}$. For every $m=1,2, \ldots$ there exists a continuous function $g$ such that $\left(g, f_{m}\right)=a_{m} \neq 0$. In the space $L^{2}(X, B, \mu)$ we can write $g$ in the form

$$
g=\sum_{n=1}^{\infty} a_{n} f_{n} .
$$

Applying $U_{\varphi}^{k}$ to the last equality, where $k$ is a fixed positive integer, we obtain

and hence

$$
V_{\mathrm{q}}^{k} g=\sum_{n=1}^{\infty} a_{n}{ }^{2 k} f_{n}
$$

$$
\lambda_{m}^{-k} U_{q}^{k} g=a_{m} f_{m}+\sum_{\substack{n=1 \\ n \neq m}}^{\infty} a_{n}\left(\lambda_{n_{t}} \lambda_{m}^{-1}\right)^{k} f_{n} .
$$

By assumption the measure $\mu$ is ergodie and so $\lambda_{n} \neq \lambda_{m}$ for $n \neq m$. Therefore

$$
\frac{1}{p} \sum_{k=0}^{p-1} \lambda_{m}^{-k} U_{q}^{k} g=a_{m} f_{m}+\sum_{\substack{n=1 \\ n \neq m}}^{\infty} a_{n} \frac{1}{p} \frac{1-\left(\lambda_{n} \lambda_{m}^{-1}\right)^{p}}{1-\lambda_{n} \lambda_{m}^{-1}} f_{n} .
$$

Since $\left|\lambda_{m}\right|=1$ and the sequence $\left(\dot{U}_{\varphi}^{k} g\right)$ is $\mathrm{w}^{*}$ cc, the sequence $\left(\lambda_{m}^{-k} U_{\varphi}^{k} g\right)$ is also a $\mathrm{w}^{*}$ ce sequence. We set $\lambda_{m}^{-1} U_{\varphi}=T$. In view of the remark in [2] on the p. 435 the arithmetic means

$$
A_{p} g=\frac{1}{p} \sum_{k=0}^{p-1} T^{k} g
$$

are also weakly * conditionally compact.

We shall prove that $\left(A_{p} g\right)$ is weakly* convergent for every $g \in C(X)$.

Let $E$ be the space of all bonnded functions $f: X \rightarrow R$ of the first Baire class with norm $\|f\|=\sup _{x \in \bar{X}}|f(x)|$.

From now on we proceed as in the proof of Theorem 3, Ch. 7.3 of [2]. Let $E_{1}=\widehat{(I-T) E}, E_{2}=\{g \in E:(I-T) g=0\}$, and $E_{3}=\left\{\xi \in E^{*}\right.$ : $\left.\left\{I-T^{*}\right) \xi=0\right\}$. 
We prove

(a) $B_{1} \cap E_{2}=\{0\}$.

Suppose now that $\left(A_{p} g\right)$ is not weakly* convergent for a certain $g \in C(X)$. Since $\left(A_{p} g\right)$ is weakly* conditionally compact, there exist two sequences of integers $\left(n_{i}\right)$ and $\left(m_{i}\right)$ such that $A_{n_{i}} g \underset{w^{*}}{\rightarrow} h_{1}, A_{m_{i}} g \underset{w^{*}}{\overrightarrow{2}} h_{2}, h_{1} \neq h_{2}$. of course, $h_{1}, h_{2} \in E$. Next we prove

(b) $h_{1}, h_{2} \in E$, which in view of (a) implies that $h_{1}-h_{2} \notin E_{1}$.

Then there exists a linear functional $\xi \in E^{*}$ such that $\xi / E_{1}=0$ and $\xi\left(h_{1}-h_{2}\right) \neq 0$. Thus $\xi((I-T) g)=0$ for every $g \in E$, which gives $\left(I-T^{*}\right) \xi$ $=0$; i.e. $\xi \in E_{3}^{*}$. Therefore

$$
\lim _{p} \xi\left(A_{p} g\right)=\lim _{p}\left(A_{p}^{*} \xi\right)(g)=\xi(g)
$$

which implies that

$$
\xi\left(h_{1}\right)=\lim _{i} \xi\left(A_{n_{i}} g\right)=\lim _{i} \xi\left(A_{n_{i}} g\right)=\xi\left(h_{2}\right) .
$$

This contradicts the choice of the functional $\xi$.

Letting $p \rightarrow+\infty$ in (1), we get

$$
h_{m}=a_{m} f_{m},
$$

so $f_{m}$ is equivalent to $a_{m}^{-1} h_{m}$, which is of the first Baire class.

COROLIARY 1. For every continuous function $g$ the limit

$$
\lim _{p} \frac{1}{p} \sum_{k=0}^{p-1} \sigma_{p}^{k} g(x)=h(x)
$$

exists and the function $h$ satisfies the following equation: $\circ \varphi(x)=h(x)$ for every $x \in X$. Hence we can choose $f_{n}$ in such way that $U_{\varphi} f_{n}(x)=\lambda_{n} f_{n}(x)$ for every $x \in X$.

The corollary follows immediately from the proof of Theorem 1 .

CoRollart 2. Suppose now that $X_{0} \subset X$ is a minimal set for a $\mathrm{w}^{*} \mathrm{cc}$ system $(X, \varphi)$. Then the eigenfunctions of $U$ are continuous on the set $\bar{X}_{0}$.

Proof. Suppose that an eigenfunction $f_{m}$ has a point of discontinuity $x_{0} \in X_{0}$. Then the oscillation of $f$ at $x_{0}$ is positive: $\omega\left(f_{n}, x_{0}\right)>0$. Since $X_{0}$ is minimal, the trajectory $\left(\varphi^{n}\left(x_{0}\right)\right)_{n=0}^{\infty}$ is dense in $X_{0}$, and we immediately conclude that $\omega\left(f_{n}, x\right)>0$ for every $x \in X_{0}$. But in view of Theorem 1. the function $f_{n}$ is of the first Baire class, and so it must have at least one point of continuity in every non-empty closed set, whence also in $X_{0}$.

In $[9]$ it is proved that if a system $(X, \varphi)$ is strictly ergodic and has a continuous spectrum, then the averages $\frac{1}{p} \sum_{k=0}^{p-1} \lambda^{k} U_{p}^{k} f(x)$ are uniformly convergent to zero for every $\lambda \in \boldsymbol{B}^{1}, \lambda \neq 1$, and for every $f \in O(X)$.
CoRoLuART 3. Every $w^{*} \mathrm{cc}$, minimal dynamical system $\left(X_{0}, \varphi\right)$ has a factor $(Y, \psi)$ of the following type: $Y$ is a closed subset of an abelian compact group $G, Y$ is invariant with respeet to a shift $\tau$ on the group, and $\varphi$ is the restriation of $\tau$ to $Y$.

Proof. We set

$$
\begin{gathered}
Y=\left\{y=\left(y_{n}\right): y_{n}=f_{n}(x), n=1,2, \ldots, n \in X\right\}, \\
\tau(y)=\left(\lambda_{n} y_{n}\right), \quad h(x)=\left(f_{n}(x)\right), \quad G=S^{1} \times S^{1} \times \ldots
\end{gathered}
$$

The system $(Y, \tau)$ is the h-image of the system $\left(X_{0}, \varphi\right)$.

COROLLART 4. Every minimal, $w^{*}$ ce dynamical system $\left(X_{0}, \varphi\right)$ is strictly ergodic.

Proof. Let $g \in O(X)$. In view of Corollaries 1 and 2 the function $h(x)=\lim _{p} \frac{1}{p} \sum_{k=0}^{p} \partial_{\varphi}^{k} g(x)$ is $\varphi$-invariant and continuous. Thus it has to be constant, which completes the proof.

Consider now the case where $X=S^{1}$ and $\varphi$ is a homeomorphism of the circle (see Example (ii)). If $\varphi$ has some periodic points or if $\varphi$ is conjugate to a rotation, then it is easy to construct all the eigenfunctions of $U_{\varphi}$. Consider now the case where $\varphi$ is a Denjoy homeomorphism. Let $f$ be an eigenfunction of $U_{\phi}$, and let $I=(a, b)$ be an are in $S^{1}$ which is a component of the set of all wandering points $S^{1}-\Delta$. Since there are no periodie points, the arcs $\varphi^{n}(I)$ are pairwise disjoint, and thus dist $\left(\varphi^{-n}(a), \varphi^{-n}(b)\right) \rightarrow 0$ as $n \rightarrow+\infty$. Hence

$$
\begin{aligned}
|f(b)-f(a)| & =\left|f\left(\varphi^{n}\left(\varphi^{n}(b)\right)\right)-f\left(\varphi^{n}\left(\varphi^{n}(a)\right)\right)\right| \\
& =\mid \lambda^{n}\left(f\left(\varphi^{-n}(b)\right)-\lambda^{n}\left(f\left(\varphi^{-n}(a)\right)\right) \mid \rightarrow 0\right.
\end{aligned}
$$

as $n \rightarrow+\infty$.

Therefore every eigenfunction of $U_{\varphi}$ has the same values at the endpoints of the components of the set $S^{1}-\Delta$. Let $h$ be a Cantor function of $\Delta$, i.e. $h$ maps $S^{1}$ onto $S^{1}$, and $h$ is continuous and constant on every component of the set $S^{1}-\Delta$. It is easy to see that $h$ also maps $\Delta$ onto $\mathcal{S}^{1}$. The map $\varphi$ induces a map $\tilde{\varphi}$ on $h(\Delta)=S^{1}: \varphi(h(x))=h(\varphi(x)), x \in \Delta$. It is east to see that $\tilde{\varphi}$ is conjugate to a rotation of $\mathcal{S}^{\mathrm{I}}$, and so all the eigenfunctions $\tilde{f}_{n}$ of $U_{\tilde{\varphi}}$ are of the form $\tilde{f}_{n}(z)=\left(\left.k\langle z)\right|^{n}\right.$, where $z \in B^{1}, k$ conjugates $\tilde{\varphi}$ and the rotation. Since every eigenfunction $f_{n}$ of $U_{q}$ takes the same values at the end-points of the components of $S^{1}-\Delta$, there exists a function $g_{n}$ on $h(A)$ such that $f_{n}(x)=g_{n} \circ h(x), x \in S^{1}$. Hence $f_{n}$ are of the form $f_{n}(x)$ $=(k \circ h(x))^{n}$. Denote by $E$ the subspace of $G(\Lambda)$ spanned by all the eigenfunctions of $f_{n}$ restricted to $\Delta$. In view of Kadec's result (see [7], Oorrolaries 9.12 and 2.3 ) the subspace $E$ has no closed complement in $O(A)$.

$K$. Deleeuw and I. Glicksberg [1] have shown that every wec dynamical system $(X, \varphi)$ has the following property: the space $C(X)$ can be 
decomposed into the direct sum of two closed subspaces $E$ and $F$, where $E$ is the subspace spanned by all the eigenfunctions of $U_{\varphi}$. So we have the following

Remaxk 1. The Deleeuw and Glicksberg result cannot be extended onto the class of $\mathrm{w}^{*} \mathrm{cc}$ mappings.

3. The $w^{*} c c$ dynamical systems seem to be so close to isometries and so similar to homeomorphisms of the circle, that one could expect that every sequence entropy (for the definition see [3]) of a $\mathrm{w}^{*} \mathrm{cc}$ mapping is equal to zero. But this is not true.

In this section we shall present an example of a $w^{*} c c$ dynamical system for which a sequence entropy is positive. The space $X$ will be chosen as a closed subset of the space of all zero-one sequences, invariant with respect to the left-side shift; the map $\varphi$ will be the shift.

Let $n \geqslant 0$ be a fixed, positive integer and let $r_{n}$ and $l_{n}$ be two nonnegative integers such that $r_{n}=2^{n} l_{n}$. Consider the family of all sequences $v_{n}=\left(v_{1}, v_{2}, \ldots, v_{r_{n}}\right)$ where $v_{k}=0$ for $k \neq i z_{n}$ and $v_{k}$ is arbitrary for $k=i i_{n}$ (i.e. $v_{l k}$ is either 0 or 1 ). Obviously there are $2^{2^{n}}$ sequences $v_{n}$. Denote them by $v_{n}^{(1)}, v_{n}^{(2)}, \ldots, v_{n}^{\left(2^{2}\right)}$. Let $\nabla_{n 2}$ be the sequence formed by all $\boldsymbol{v}_{n}^{(i)}$ written in one row:

Now we set

$$
\nabla_{n}=v_{n}^{(1)} v_{n}^{(2)} \ldots v_{n}^{\left(2^{2}\right)}
$$

$$
x_{0}=\nabla_{0} \nabla_{1} V_{2} \ldots \nabla_{n} \ldots \in\{0,1\}^{Z} .
$$

We have to define the integers $l_{n}$. Let $\boldsymbol{v}_{n_{0}}^{(1)}=(0), v_{0}^{(2)}=(1)$, i.e. $l_{0}=1$, $r_{0}=1$. We require that $l_{n}$ should be greater that the length of the sequence $V_{0} V_{I} V_{2} \ldots V_{n-1}$. Since the length of $V_{i}$ is equal to $2^{2^{i}} r_{i}$, the number $l_{n}$ has to be greater than $\sum_{i=0}^{n-1} 2^{2^{i}} r_{i}$.

So we set

$$
l_{n}=n \cdot 2^{2^{n}} r_{n-1}=n 2^{2^{n}+n} l_{n-1} .
$$

Denote the coordinates of $x_{0}$ by $\xi_{0}, \xi_{1}, \ldots$ Let $p_{n}$ be an integer such that

$$
\left(\xi_{0}, \ldots, \xi_{p_{n}}\right)=\nabla_{0} \nabla_{1} \ldots V_{n} \text { for } n=0,1, \ldots
$$

In other words, $p_{n}-p_{n-1}=$ length of $\nabla_{n}$.

Denote $x_{n}=\varphi^{n}\left(x_{0}\right)$ and set $X=$ closure $\left\{x_{n}, n=0,1, \ldots\right\}$.

Proposition 3. The space $X$ contains only points of the form (i) $x_{n}$, $n=0,1, \ldots$, (ii) $e_{n}=(0,0, \ldots, 1,0, \ldots), n=0,1, \ldots$, (iii) $(0,0, \ldots)$.

Proof. Suppose $x=\left(\eta_{0}, \eta_{1}, \ldots\right)$ is a cluster point of the set $\left\{x_{n}\right\}$. Then there exists a sequence $\left(x_{n_{k}}\right), n_{k}<n_{k^{+}+1}$, such that $x_{n_{k}} \rightarrow x$. Suppose that $\eta_{m}=1$ for an index $m$. Thus for $k$ large enough the $m$ th coordinates of $x_{n k}$ have to be 1 . Let $i \neq m$ be fixed. Since $l_{n} \rightarrow+\infty$, all the coordi- nates of $x_{n_{k}}$ have to be zero for $k$ large enough. Hence $\eta_{i}=0$ for arbitrary $i \neq m$ and $e_{m}=x \in X$. Since $e_{m} \rightarrow(0,0, \ldots)$, the point $(0,0, \ldots)$ also belongs to $X$.

CoRoLlaRT 5. The system $(X, \varphi)$ is w*c.

Indeed, by Proposition 3 the space $X$ is denumerable, and so every $\operatorname{map}$ of $X$ is $\mathrm{w}^{*} \mathrm{cc}$.

PRopositron 4. The sequence entropy of the system $(X, \varphi)$ is positive.

Proof. Let $A_{n}=\bigcup_{i=0}^{n}\left\{p_{i}+s l_{i}\right\}_{s=1}^{i}$. Then $\operatorname{Card} A_{n}=\sum_{i=0}^{n} 2^{i}=3^{n+1}-1$. We set $A=\bigcup_{n=0}^{\infty} A_{n}$ and we choose an open cover of $X$ in the standard way: $a=\left(\left\{x=\left(\zeta_{i}\right): \zeta_{0}=0\right\},\left\{x=\left(\zeta_{i}\right): \zeta_{0}=1\right\}\right)$

(It is also a partition of $X$.) In view of the definition of $v_{n}, \nabla_{n}$ and $l_{n}$ it is easy to check that the cover $a_{n}=\bigvee_{k \in A_{n}} \varphi^{-k}(a)$ contains at least as many sets as the cardinality of the set of all $v_{n}$, i.e.

$$
\operatorname{Card} \underset{k \in A_{n}}{\bigvee} p^{-k}(a) \geqslant 2^{2^{n}} \text {. }
$$

Thus

$$
\frac{1}{\operatorname{Card} A_{n}} \log N\left(a_{n}\right) \geqslant \frac{1}{2^{n+1}-1} \log 2^{2^{n}}=\frac{2^{n}}{2^{n+1}-1} \log 2 .
$$

Hence

$$
h_{-4}(\varphi, a)=\lim _{n} \sup \frac{1}{\operatorname{Card} A_{n}} \log N\left(a_{n}\right) \geqslant \frac{1}{2} \log 2 .
$$

The example presented above gives us the following

CoRouxARY 6. The sequence entropy cannot be attained on the set of non-wandering points.

Remark 2. The example presented shows also that the supremum of all measure sequence entropies is not equal to the topological sequence entropy. This result has been observed by Goodman [3]. This example is different from that of Goodman.

\section{References}

[1] K. Deleecw, I. Glicksberg, Applications of almost periodic compactifications, Acta Math. 105 (1961), pp. 63-67.

[2] N.Dunford, J. T. Schwartz, Linear operators I, Intersoience Publ, INC, New Fork 1967.

[3] T. N. T. Goodman, Topological sequence entropy, Proc. Lond. Math. Soc. (3) 29 (1974), pp. $331-350$.

[4] [A. G. Kushnirenko] А. Т. Кушниренк 0,0 метрияеских инвариантах munа әнтропии, У.M.H. 22 (5) (1967), pp. 57-65.

[5] Z. Nitecki, Differentiable dynamics, MIT Press 1971

[6] F. Odell, H. P. Rosenthal, Bunach spaces containing $l^{1}$, Israel J. Math. 20 (1975), pp. 375-384. 
[7] A. Pełezyński, Linear extensions, linear averagings, and their applicalions to the linear topological olassification of spaces of oontimuous funotions, Dissertationes Mathematicae 58, PWN 3968.

[8] H. P. Rosenthal, A charaeterization of Banaeh spaces containing $l^{1}$, Proc. Nat. Acad. Sci. USA $71(6)$, pp. 2411-2413.

[9] B. Sehmitt, Théoreme ergodique ponetuel pour les suites uniformes, Ann. Inst. H. Poincaré 8 (4) (1972), pp. 387-394.

[10] R. Sine, Convergence theorems for weakly almost periodie Markov operations, Israel J. Math, 19 (1974), pp. 246-255.

[11] S. Smale, Differentiable dynamical systems, Bull. Amer. Math. Soc. 73 (1967), pp. 747-817.
The algebra of compact operators does not have

any finite-codimensional ideal

by

\author{
PIERRE DE LA HA RPE (Genóve)
}

Abstract. The Lie algebra of compact operators on an infinite dimensional Hilbert space has no non-trivial finite-dimenzional quotient. It follows that, as a Banach-Lie algebra, all its extensions are trivial.

Let $H$ be an infinite-dimensional real or complex Hilbert space and let $C(H)$ be the algebra of all compact operators on $H$. It is well known that any non-trivial two-sided ideal of $O(H)$ contains the ideal of all finite rank operators (Theorem 1.7 in [2]); the standard examples are the von Neumann-Schatten ideals [5]. The purpose of this note is to show that any such ideal has infinite codimension. We shall indeed prove the following stonger statement, which is phrased in the Lie algebra setting; as a corollary we obtain that any extension (in a suitable sense) of the Lie algebra considered is trivial.

THEOREM. Let $g \boldsymbol{l}(H, C)$ be the Lie algebra defined by the commutator product on $O(H)$ and let $\boldsymbol{a}$ be a non-trivial ideal in $\boldsymbol{g l}(H, O)$. Then a contains the space $\mathrm{sl}\left(H, O_{0}\right)$ of all finite rank operators with zero trace and $a$ has infinite codimension.

That $a$ contains $s l\left(H, C_{0}\right)$ is an easy corollary of Schur's lemma and of the simplicity of the Lie algebra $s l\left(H, C_{0}\right)$; see for example [3], page I.2. For the second statement, consider a finite-dimensional Lie algebra $g$, say, of dimension $d>0$, and a morphism $\pi: \boldsymbol{g l}(\boldsymbol{H}, O) \rightarrow \boldsymbol{g}$. We have to show that the kernel of $\pi$ is $g l(H, C)$ itself. We do this below in the complex case; the real case will follow from a standard complexification argument.

We denote by $\boldsymbol{N}$ the set of natural integers including zero and by $c_{\mathrm{o}}$ the space of sequences $\left(x_{n}\right)_{n \in \mathbf{N}}$ of complex numbers converging towards zero.

Limara 1. Let $H_{0}$ be a closed subspace of $H$ of infinite dimension and infinite codimension. Let $\left(e_{n}\right)_{n \in N}$ be an orthonormal basis in $H_{0}$ and let $\left(x_{n}\right)_{n \in N}$ 